\title{
Comparative evaluation of PCO development in various methods of cataract surgeries and with different type of IOL implantation
}

\author{
Swathi Pinnamaneni ${ }^{1}$, Sandeep. ${ }^{2, *}$, M. S. Usha ${ }^{3}$ \\ ${ }^{1}$ Consultant, ${ }^{3}$ Chief Consultant \& HOD, Dept. of Ophthalmology, Mysore Race Club Eye Hospital, Mysore, Karanataka, \\ ${ }^{2}$ Associate Professor, Dept. of Ophthalmology, P K Das Institute of Medical Sciences, Medical College Hospital, Kerala, India
}

*Corresponding Author:

Email: drsandeepsms@gmail.com

\begin{abstract}
Aims: 1 . To clinically evaluate posterior capsular opacification in various types (ECCE, SICS, Phacoemulsifiction) of cataract surgeries; 2 . To clinically evaluate posterior capsular opacification in various types of IOL implantation.

Materials and Methods: The source of the data included, Patients with posterior capsular opacification attending the ophthalmic outpatient department after various types of cataract surgeries with various types of IOL implantation. The present study is a hospital based prospective study conducted from October 2012 to April 2014. The duration of the study was one year six months and follow up period of one month.

Results: In this hospital based prospective study 219 patients with posterior capsular opacification were enrolled during a study period of 1 year and 6 months. Out of these 219 patients 200 patients were included in the study. 19 patients were excluded from the study. Out of 19 patients excluded from the study, 3 patient had PCO in post vitrectomised eyes, 3 had macular scars and 4 patient had subluxated IOLs, 4 patients had diabetic CSME and 5 patients were lost for follow up.

Conclusion: Posterior capsular opacification was found more in patients undergoing small incision cataract surgery with rigid intraocular lenses.

Incidence of PCO is more in females compared to males; In the bag IOL implantation had a reduced incidence of PCO; Single piece IOLs are more commonly associated with posterior capsular opacification.
\end{abstract}

Keywords: Intraocular lens, PCO, Phacoemulsiation, SICS.

\section{Introduction}

Cataract surgery has come a long way from couching to phacoemulsification with foldable intraocular lens implantation. ${ }^{1}$ Advances in surgical techniques, intraocular lens materials, and designs have transformed outcomes in cataract surgery. However, posterior capsular opacification (PCO) remains one of the most common postoperative morbidity. ${ }^{2}$

The incidence of posterior capsular opacification rate quoted by David $\mathbf{J}$ Apple who is a pioneer in pathology of intra ocular lens opacification ranges from $1.3 \%$ to $14.6 \% .^{3}$

In eyes undergoing cataract surgery for uncomplicated senile cataract the incidence of PCO is known to range from as high as $50 \%$ to as low as $<5 \%{ }^{6}$

Posterior capsularopacification is a multifactorial physiological consequence of cataract surgery. The proliferation, migration and abnormal differentiation of residual lens epithelial cells and fibers in the capsular bag have been implicated in the pathogenesis of posterior capsule opacification (PCO). ${ }^{4}$

\section{Aims and Objectives}

1. To clinically evaluate posterior capsular opacification in various types (ECCE, SICS, Phacoemulsifiction) of cataract surgeries

2. To clinically evaluate posterior capsular opacification in various types of IOL implantation

\section{Materials and Methods}

The source of the data included:

1. Patients with posterior capsular opacification attending the ophthalmic outpatient department at Mysore Race Club Eye Hospital, presenting with diminution of vision.

2. The present study is a hospital based prospective study conducted in Mysore Race Club Eye Hospital from October 2012 to April 2014. The duration of the study was One year six months and follow up period of one month.

\section{Inclusion Criteria}

1. Patients aged $>40$ years with posterior capsular opacification who underwent cataract surgery for senile/traumatic/complicated cataract.

2. PCO in patients with established glaucoma/uveitis under control.

3. PCO in patients post panretinal photocoagulation/vascular diseases.

4. Patients with established myopia in whom IOL was inserted under guarded visual prognosis.

\section{Exclusion Criteria}

1. Patients with PCO aged $<40 \mathrm{yrs}$.

2. postvitrectomised eye

3. Post retinal detachment surgery.

4. PC rent with or without vitreous loss.

5. Subluxated IOLs.

A total of 200 eyes of 193 patients were included in the study as per above mentioned inclusion and exclusion criteria. All the patients were evaluated by taking detailed history with special reference to the 
duration from cataract surgery, type of surgery, type of Intra ocular lens implanted, any complication of surgery, visual recovery after surgery and any associated systemic illnesses such as diabetes and hypertension.

\section{Observations and Results}

In this hospital based prospective study 219 patients with posterior capsular opacification were enrolled during a study period of 1 year and 6 months. Out of these 219 patients 200 patients were included in the study. 19 patients were excluded from the study. Out of 19 patients excluded from the study, 3 patient had PCO in post vitrectomised eyes, 3 had macular scars and 4 patient had subluxated IOLs, 4 patients had diabetic CSME and 5 patients were lost for follow up.

Statistical Analysis Used: To analyze the data following statistical methods were used in the study:

Descriptive statistics

Contingency table analysis (Cross tabs)

Chi-square test

One-Way ANOVA test

Paired samples $t$ test

All the statistical calculations were done through SPSS for windows.

The $\mathrm{p}$ value $<0.05$ was taken as statistically significant (v 16.0). The observations and results of the study are depicted in the form of tables and their respective graphs as shown below.

Age Distribution: In this present study all 200(100\%) patients in the study group were in the age group of 40 years to 95 years and with the mean age being $66.95 \pm$ 10.31years

Table 1: Diminution of vision due to posterior capsular opacification

\begin{tabular}{|l|c|c|}
\hline Duration of DOV & No. of cases & Percentage \\
\hline$<1$ mon & 19 & 9.5 \\
\hline $1-3$ mon & 70 & 35.0 \\
\hline$>3$ mon & 111 & 55.5 \\
\hline Total & 200 & 100 \\
\hline
\end{tabular}

In the present study out of 200 patients vision in $62(31 \%)$ was $<6 / 60$ and in $100(50 \%)$ patients it was $6 / 60$ to $6 / 18$, and in 38 patients it was $\geq 6 / 12$. Majority of the patients in the study group had poor visual acuity at the time of presentation. This observation is statistically significant (P 0.0001)

Table 2: Grading of posterior capsular opacification $^{5}$

\begin{tabular}{|l|c|c|}
\hline Grading of PCO & No. of cases & $\begin{array}{c}\text { Percentage } \\
\text { of cases \% }\end{array}$ \\
\hline Grade 0 & 0 & 0 \\
\hline Grade $1+$ & 32 & 16 \\
\hline
\end{tabular}

\begin{tabular}{|l|l|l|}
\hline Grade 2+ & 96 & 48 \\
\hline Grade 3+ & 72 & 36 \\
\hline
\end{tabular}

Shows the grading of posterior capsular opacifiaction in the study group

In the present study out of 200 patients none of the patients in the had grade $0 \mathrm{PCO}, 32(16 \%)$ patients had grade $1+$ PCO, $96(48 \%)$ had grade $2+\mathrm{PCO}, 72(36 \%)$ had grade 3+ PCO. Majority of the patients in the study group had grade $2+$ and grade $3+$ PCO which is correlating with the decreased visual acuity at the time of presentation.

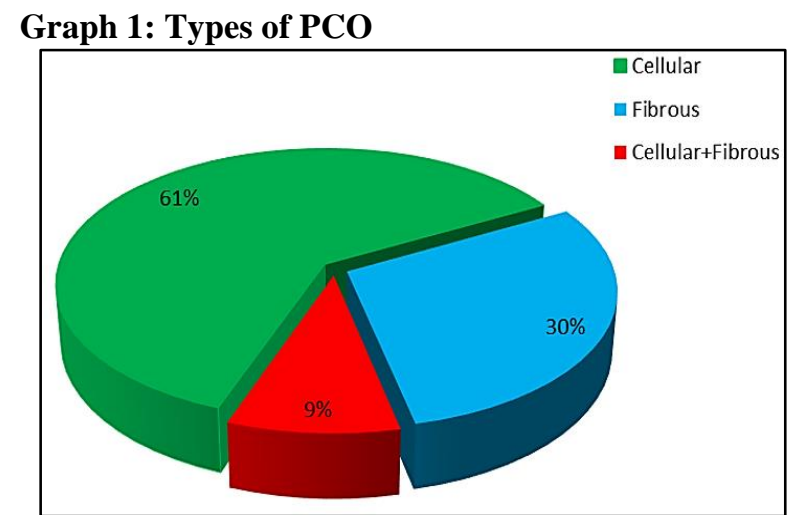

In this study out of 200 cases, $123(61.5 \%)$ patients had cellular PCO, 59(29.5\%) had fibrous, 18(9\%) had cellular and fibrous PCO. Majority of patients in the present study had cellular PCO.

In the present study further analysis was made depending on the nature of the PCO where ever it is relevant.

It is observed that, mean age in the study is $66.95 \pm$ 10.31years. No statistically significant age difference was found amongst any of the groups.

Type of Cataract Surgery: In this study out of 200 patients, 158(79\%) had undergone SICS, 39 19.5\%) phacoemulsification, $3(1.5 \%)$ ECCE. Majority of patients had undergone SICS surgery which was statistically significant $p<0.0001$. This significant number of patients in SICS group could be because of the cost effectiveness of SICS procedure. 


\section{Graph 2: Material of IOL Implanted}

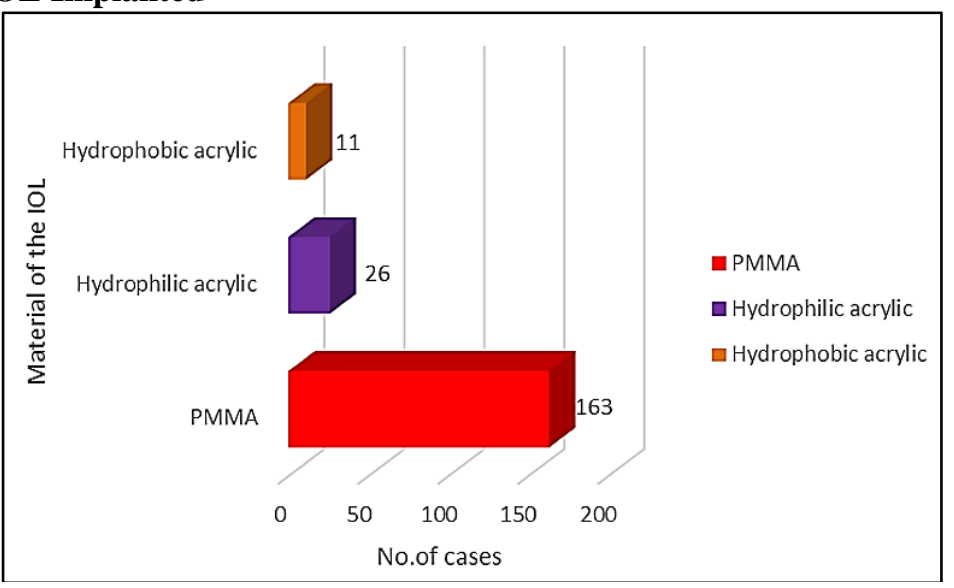

Out of 200 cases in the study group 163(81.5\%) had PMMA IOL implanted, 26(13\%) had hydrophilic acrylic IOL implanted, and in $11(5.5 \%)$ patients hydrophobic acrylic IOL was implanted. This majority of the patients in the present study group implanted with PMMA IOL which is statistically significant $\mathrm{p}<$ 0.012 . This significant number is could be because majority of the patients in the study had under gone SICS surgery and the IOL implanted in the SICS is PMMA IOL.

\section{Graph 3: Types of IOL}

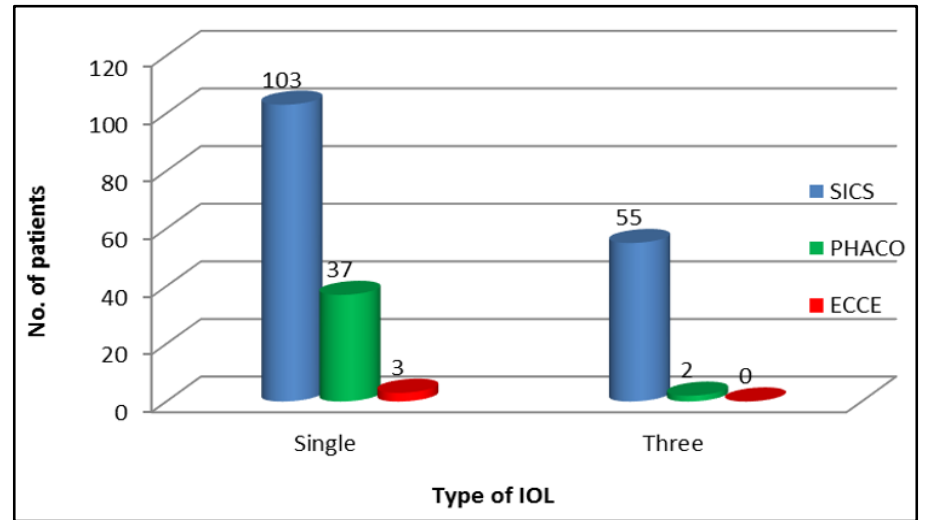

In the present study, out of 143(100\%) single piece IOLs, 103(72\%) single piece IOLs were noted in SICS, $37(25.9 \%$ in phacoemulsification group and $3(2.1 \%)$ in ECCE group. Out of $57(100 \%)$ three piece IOLs $55(96.5 \%)$ were noted in SICS group, $2(3.5 \%)$ in phacoemulsification group and $0(0 \%)$ in ECCE group. In the present study majority of the patients are implanted with single piece IOLs. Out of 200 patients, in $116(58 \%)$ the IOL is placed in the bag where as in 84 $(42 \%)$ patients it was places in the sulcus. All the 84 sulcus placed IOLs in the study were seen in the SICS and this finding shows $5.16 \%$ patients in SICS group had IOLs in the sulcus. None of the patients in phacoemulsification group had in the sulcus IOLs.

\section{Discussion}

Posterior capsule opacification (PCO) is the most common sequelae following intra ocular lens implantation surgery, occurring months to years after cataract surgery and decreasing vision significantly. Nd: YAG laser is the most modern tool to treat posterior capsular opacification and has high success rate.

The present study has been under taken to analyze probable causative factors in each case such as nature of IOL material, position of IOL-whether in the bag/in the sulcus etc and to evaluate the visual outcome following YAG laser posterior capsulotomy. This study was done in Mysore Race Club Eye Hospital between October 2012 to April 2014.

In this study out of 200 patients the mean age of patients presenting with $\mathrm{PCO}$ was $66.95 \pm 10.31$ years. This is similar to the study conducted by Gustavo et $\mathrm{al}^{6}$ (53 eyes) where the mean age was 67 years. In a study conducted by Hassan Hashemi et $\mathrm{al}^{7}$ (558 eyes) mean age was $67.0 \pm 8.9$ years and a study done by Ajite et $\mathrm{al}^{8}$ (109 eyes) mean age presentation of PCO was $67.8 \pm 8$ years. Hence mean age in the present study, is comparable with the other studies. This is perhaps the age at which patients with age related cataract present to the Ophthalmologist due to visual incapacitation and undergo cataract surgery. 
In this study, incidence of PCO was high in females. Out of 200 patients with PCO 93(46.2\%) were males and $107 \quad(54.8 \%)$ were females. Similar observations were seen in the studies by Mohan Lal Gupta et $\mathrm{al}^{9}$ (49\% were males and $51 \%$ females) Aurangzeb Shaikh et $\mathrm{al}^{10}(45.55 \%$ males and $54.45 \%$ females) Hiroshi Ando et $\mathrm{al}^{11}$ (males 34\%, females $65.2 \%)$.

In the present study, out of 200 patients, duration since cataract surgery to Nd-Yag laser capsulotomy in $11(5.5 \%)$ cases was less than 12 months, $158(79 \%)$ cases was 13 to 60 months $25(12.5 \%)$ cases was 60 to 120months, 6(3\%) was greater than 120 months which was similar to study done by Mahtabak et al ${ }^{12}$ where 75\% patients developed PCO between 24 to 48 months.

In the present study the mean time interval from cataract surgery to Nd-Yag laser capsulotomy in different types of PCO was $43.54 \pm 31.34$ months. Similar observations were made in the studies by Meenazaidi et $\mathrm{al}^{13}$ (31.10 months) Hassan Hashemi et $\mathrm{al}^{6}$ (32.8 \pm 15.8 months). The mean time interval from cataract surgery to Nd-Yag laser capsulotomy in the present study is little higher compared to other studies and this can be attributed to good vision in the other eye or inaccessibility to the hospital.

In the present study 53(26.5\%) patients had diabetes. The incidence of PCO and time interval from cataract surgeryto Yag-laser capsulotomy did not show a significant difference between diabetic and nondiabetic individuals. This was similar to a study done by done Hassan Hashemi et $\mathrm{al}^{6}$ in 2012, in which out of 558 patients $21.6 \%$ were diabetics.

In this study out of 200 patients, 158(79\%) had undergone SICS, 39 (19.5\%) phacoemulsification,
$3(1.5 \%)$ ECCE. The reason for higher number of SICS cases in the present study might be because as SICS is a cost effective procedure majority of the patients under go SICS. All the patients with SICS are implanted with PMMA lens and it is known that the incidence of PCO is higher in PMMA material compared to other IOLs. In phacoemulsification surgery acrylic IOLs are implanted which is said to cause lower PCO rate. IOLs are implanted in the bag in the phacoemulsification surgery so less number of cases in the phacoemulsification group. This finding is similar to the study done by Muhammad Moin et $\mathrm{al}^{14}$ where $6 \%$ of patients with PMMA IOL developed PCO compared to $1.5 \%$ patients with acrylic hydrophobic IOLs. This finding in this study is also similar to study done by Col PS Moulick et $\mathrm{al}^{1}$ where $21.7 \%$ of ECCE group, $13.1 \%$ of SICS group, $9.1 \%$ of phacoemulsification group developed PCO.

Out of $158(100 \%)$ cases of SICS, in 103(65.18\%) of the cases and $3(100 \%)$ of ECCE the IOL implanted was single piece PMMA and out of $39(100 \%)$ cases of phacoemulsification $37(94.87 \%)$ were single piece acrylic. This shows that the incidence of PCO was higher in single piece this finding. Is similar to the study done by Hiroshi Ando et $\mathrm{al}^{11}$ done in 2003 where the relative risk was significantly higher in single piece PMMA than three piece PMMA and he also opined that it may be related to IOL surface and edge designs which could be the same in the present study. In present study, cellular PCO was the predominant type of PCO $123(61.55 \%)$ cases, fibrous PCO59 (21.5\%) cases, cellular-fibrous 18 (9\%) cases. This finding is similar a study done by Mohan Lal Gupta et $\mathrm{al}^{9}$ (2012).

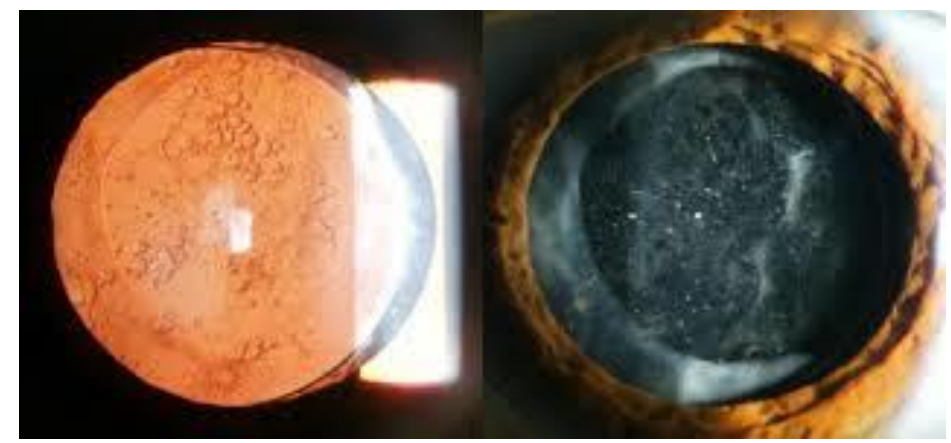

Fig. 1: Picture showing cellular PCO, fibrous PCO

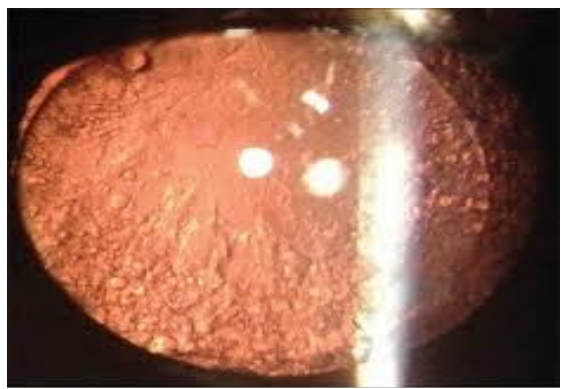

Table 3: Comparision of types of PCOs with similar studies

\begin{tabular}{|l|c|c|c|c|}
\hline \multicolumn{1}{|c|}{ Type of PCO } & \multicolumn{2}{c|}{$\begin{array}{c}\text { Mohan lal } \\
\text { Guptha et al } \\
\text { (100 eyes) }\end{array}$} & \multicolumn{2}{c|}{$\begin{array}{c}\text { Present study } \\
\text { (200 eyes) }\end{array}$} \\
\cline { 2 - 5 } & No. & $\%$ & No. & $\%$ \\
\hline Cellular & 65 & 65 & 123 & 61.55 \\
\hline Fibrous & 35 & 35 & 59 & 21.5 \\
\hline Cellular+Fibrous & - & - & 18 & 9 \\
\hline
\end{tabular}

Fig. 2: Cellular-fibrous type of PCO 
This finding that cellular PCO common in the present study is in contrast to study done by Bhargava et $\mathrm{al}^{15}$ where out of 215 patients $40(18.6 \%)$ had cellular PCO,103 (47.5\%) had fibrous PCO,72 (33.48\%)had cellular-fibrous PCO.

In the present study group, $80.5 \%$ cases underwent SICS and ECCE surgery in which the implanted IOL was PMMA material, of which $51.5 \%$ were placed in the sulcus. The remaining $19.5 \%$ of the cases underwent phacoemulsification, in which all the IOLS were implanted in the bag. This is similar to the study done by Meacock et $\mathrm{al}^{16}$ (2001).

In SICS group, $51.5 \%$ of the IOLs were implanted in the sulcus and in the remaining $48.5 \%$ of the cases though the IOL was placed in the bag, the continuous curvilinear capsulorrhexis was larger than the optic size. This explains the higher occurrence of PCO in PMMA IOLs in the study group both in the sulcus and in the bag. Whereas smaller continuous curvilinear capsulorrhexis and the nature of the IOL material justify the occurrence of PCO in only $19.5 \%$ of the phacoemulsification group which is less than the SICS group.

\section{Conclusion}

Posterior capsular opacification is the most common cause for decrease in visual acuity following cataract surgery.

1. Majority of patients with PCO presented 1-5 years (13- 60 months) after cataract surgery.

2. Incidence of $\mathrm{PCO}$ is more in females compared to males

3. Patients who underwent small incision cataract surgery with PMMA IOL had higher rate of occurrence of PCO. Posterior capsular opacification was more common in patients with single piece IOL as compared to three piece IOL.

\section{References}

1. Col PS Moulick, Col FEA Rodrigues, et al. Evaluation of posterior capsular opacification following phacoemulsification, Extracapsular and small incision cataract surgery. MJAFI. 2009;65(3):225-228.

2. Niranjan Awasthi, Suqin Guo, et al. Posterior Capsular Opacification A Problem Reduced but Not Yet Eradicated, Arch Ophthalmol. 2009;127(4):555-562.

3. David J Apple. Influence of intraocular lens material and design on postoperative intracapsular cellular activity, Trans Am Ophthalmol Soc. 2000;98:257-283.

4. Suresh K Pandey, David J Apple, Liliana Wermer, et al. Posterior capsule opacification: A Review of the Aetiopathogenesis, Experimental and clinical studies and factors for prevention. Indian J Ophthalmol. 2004;52(2):99112.

5. Usha Koul Raina, Gupta Vinita, Mehta DK. PCCC in pediatric cataract with and without optic capture in absence of vitrectomy: a prospective randomized study. AIOS 2004;1-4.

6. Montenegro GA, Marvan P, Dexl A, Picó A, et al. Posterior Capsule Opacification Assessment and Factors That Influence Visual Quality After Posterior Capsulotomy, Am J Ophthalmol. 2010;150(2):248-253.
7. Hassan Hashemi, S-Farzad Mohammadi, et al. Posterior Capsule Opacification after Cataract Surgery and its Determinants Iranian Journal of Ophthalmology. 2012;24(2):3-8.

8. Ajite K.O., Ajayi I.A., et al. Visual outcome of Patients with Posterior Capsular Opacification Treated with Nd: YAG Laser, Journal of Medicine and Medical Research. 2013 December, 1(4):23-27.

9. Mohan Lal Gupta et al., Visual benefits of Nd: YAG laser capsulotomy study in south eastern rajasthan. Int J Biol Med Res. 2012;3(4):2507-2514.

10. Aurangzeb Shaikh, Faheemullah Shaikh, et al. Prevalence of different Nd: YAG Laser induced complication in patients with significant posterior capsule opacification and their correlation with time duration after standard cataract surgery. International Journal of Medicine and Medical Sciences. 2010 January, 2(1):12-17.

11. Hiroshi Ando, Nobuyo Ando, Tetsuro Oshika. Cumulative probability of neodymium: YAG laser posterior capsulotomy after phacoemulsification. J Cataract Refract Surg. 2003;29(11):2148-2154.

12. Mahtab Alam Khanzada, Shafi Muhammad Jatoi, et al. Is the Nd: YAG Laser a Safe Procedure for Posterior Capsulotomy, Pak J Ophthalmol. 2008;24(2):73-78.

13. Meena Zaidi, Saiyid Nasir Askari. Effect of Nd-Yag laser capsulotomy on anterior chamber depth, intra ocular pressure and refractive status, Asian J Opthalmol. 2004;5(4):2-5.

14. Muhammad Moin, Kashif Raza, Anwar Ul-Haq Ahmad. Posterior Capsular Opacification after PMMA and Hydrophobic Acrylic Intraocular Lens Implantation, Pak J Ophthalmol. 2009, 25(4).

15. Bhargava R, Kumar P, Prakash A, Chaudhary Estimation of mean ND: Yag laser capsulotomy energy levels for membranous and fibrous posterior capsular opacification, Nepal J Ophthalmol. 2012;4(7):108-113.

16. Meacock WR, Spalton DJ, Hollick EJ, et al. The effect of polymethylmethacrylate and acryS of intraocular lenses on the posterior capsule in patients with a large capsulorrhexis. Jpn J Ophthalmol. 2001;45(4):348-354.

How to cite this article: Pinnamaneni $\mathrm{S}$, Sandeep. K, Usha M. S., Comparative evaluation of PCO development in various methods of cataract surgeries and with different type of IOL implantation. Ind $\mathbf{J}$ Clin Exp Ophthalmol. 2018;4(3):304-308. 